\title{
Shape memory alloys: From the physical properties of metastable phase transitions to dampers for civil engineering applications
}

\author{
V. Torra ${ }^{1}$, A. Isalgue ${ }^{1}$, F.C. Lovey ${ }^{2}$, F. Martorell ${ }^{1}$, F.J. Molina ${ }^{3}$, M. Sade ${ }^{2}$ \\ and $\mathrm{H}$. Tachoire ${ }^{4}$ \\ ${ }^{1}$ CIRG-DFA-ETSECCPB-UPC C/. Gran Capità s/n, Campus Nord B-4, \\ 08034 Barcelona, Spain \\ ${ }^{2}$ Div. Metales, CAB-Instituto Balseiro, 8400 S.C. Bariloche, Argentina \\ ${ }^{3}$ ELSA Laboratory, IPSC, EC-JRC, 21020 Ispra, Italy \\ ${ }^{4}$ MADIREL/Thermochimie, UMR 6121, Université de Provence, Centre de \\ Saint-Charles, 13331 Marseille Cedex 03, France
}

\begin{abstract}
The physical properties of Shape Memory Alloys (SMA), useful in damping effects of scarce events as quakes, are studied for $\mathrm{Cu}$-based alloys (CuAlBe) and partially for $\mathrm{NiTi}$ in the appropriate time scale. The evaluation of the deformation $(\varepsilon)$ in the hysteresis cycle and in their internal loops, against the thermodynamic forces, i.e. the external stress $\left(\sigma_{\text {ext }}\right)$ and temperature $(T)$ is performed. The main goal for the applicability of the alloys centers in reliable behavior after several years of inactivity. The alloy require a minimal transformation temperature change and, also, within a reduced transient time to ensure that the material always remain inside the thermoelastic window (full parent phase recovery at zero stress and without permanent deformation at maximal stress). The macroscopic behavior associated with the Clausius-Clapeyron equation, the material fatigue and the local temperature changes, produced by latent heat and by frictional contributions, are critically visualized. The microscopic phenomena related with the transient after quenched effect, the evolution in the region of phase coexistence and the long time seasonal actions in parent phase are, also, evaluated.
\end{abstract}

\section{INTRODUCTION}

The particular properties of Shape Memory Alloys (SMA) arise from a recoverable (or "reversible") martensitic transformation so-called a first-order transition between metastable phases [1-3]. The transformation releases and absorbs latent heat and also, delivers.heat produced by frictional contributions (interface movement and nucleation). Among materials described in the literature, one can mention the Cu-based alloys [1-5] (as the CuZnAl, the CuAlNi or the CuAlBe) and also, the nickel-titanium alloys: the nitinol or NiTi [2, 4-5]. The representative coordinates of the state equation for one-stress-axis in rods or wires are the external force or the stress $\sigma$, the length or the deformation strain and the temperature. The alloy application requires the evaluation of the deformation in the hysteresis cycle and in their internal loops against the thermodynamic forces, i.e. the external stress $\left(\sigma_{\text {ext }}\right)$ and temperature $(\mathrm{T})$.

Recently, several smart or active devices have been developed to smooth the effects of the mechanical oscillations via mechanical compensation as in gondola systems i.e., in skiing facilities [6]. Also, the electrorheological or magnetorheological fluids allow to design efficient damper device with variable viscosity via the appropriate feedback of high voltage or intensity current respectively. At very long term, these fluid devices require some periodic attention and, also, an easy accessibility to assure a correct refilling and mixture homogeneity, repair and revision. To some extent, these requirements are partially inappropriate when only scarce events will be damped. In Civil Engineering it is expected a very long term guaranteed operation (i.e. several years or tens of years) without continuous attention and with reduced accessibility of integrated devices in the built system. Particularly, earthquake damping devices performing several tens of cycles at relative high frequency (i.e. $0.5 \mathrm{~Hz}$ ) and at relevant deformation (i.e. $10 \mathrm{~cm}$ or more) are expected. The main advantage of the SMA guaranteed dampers, in comparison with other damper systems, relates the appropriateness of the devices for working integrated with the structure without permanent surveillance. To ensure 
closed hysteresis cycles, without permanent plastic deformation or fracture, the external stress $\left(\sigma_{\text {ext }}\right)$ need to be situated inside the pseudoelastic window under the plastic deformation $\left(\sigma_{\mathrm{pd}}\right)$ stress: $0 \leq \sigma_{\text {ext }} \leq \sigma_{\text {pd. }}$. A guaranteed behavior of the SMA can be established throughout the experimental analysis of the macroscopic and microscopic effects on the materials that modify the hysteresis cycle position inside the pseudoelastic window according with the technical conditions. At the macroscopic level, two classical effects need to be evaluated: the direct effect of seasonal temperatures on the phase transition modifying the position of the hysteresis cycle (the ClausiusClapeyron coefficient) and the irreversible thermal effects inducing local temperature changes by the actions of latent heat and by frictional contributions. At mesoscopic level, the sample fatigue is generated by the dislocation creation on cycling. This is a non-classical phenomena induced by the interaction between martensite variants and between martensite and grain boundaries. For instance, the interactions can produce dislocations when the local stresses overcome the plastic deformation limit in the austenite or martensite. At microscopic level several atomic order effects are noticed, i.e., a long-term evolution of the atomic order in parent phase after quenching, the tracking effects of seasonal temperature changes and, also, evolutions induced by coexistence between the phases.

In this work, the conditions imposed by civil engineering on dampers [7-8] inducing forces near $30 \mathrm{kN}$ (i.e. for application in family houses), are experimentally analyzed: the hysteresis cycle and the internal loops, the Clausius-Clapeyron coefficients, the thermal effects induced by "latent" heat and frictional contributions, and the microscopic contributions induced by seasonal temperatures and phase coexistence. Some comparative analysis between CuAlBe and NiTi alloys is performed. A representative model of the SMA is established and the behavior of a SMA is simulated.

\section{EXPERIMENTAL}

$\mathrm{Cu}$-based and NiTi alloys are used. The $\mathrm{Cu}-\mathrm{Zn}-\mathrm{Al}$ single crystal samples have been homogenized at $1123 \mathrm{~K}$ during $10 \mathrm{~min}$ and subsequently quenched in water at room temperature $(293 \mathrm{~K})$. The used strips of TiNi (from the manufacturer) was submitted to aging $(1 \mathrm{~h})$ at $673 \mathrm{~K}$ in order to obtain pseudo-elastic behavior. Several polycrystalline samples of Cu-Al-Be (supplied by Trefimetaux, $\mathrm{KME}$, France) have been used with As temperature close to $263 \mathrm{~K}$, with nominal atomic composition near to $\mathrm{Al} 12 \mathrm{wt} \%, \mathrm{Be} 0.5 \mathrm{wt} \%$ and $\mathrm{Cu}$ to balance. The samples have been homogenized by means of short times at $1123 \mathrm{~K}$ ( 1 to 4 minutes for the different cross sections, between 1 to $17 \mathrm{~mm}^{2}$ ) to avoid excessive grain growth, quenched in water at $293 \mathrm{~K}$ and then immediately aged for variable times at temperatures near $373 \mathrm{~K} \mathrm{~A}$ stress-strain analysis at three different levels is done. Some work is performed using a high resolution equipment $(1 \mathrm{mN}, 0.1 \mu \mathrm{m}$ and $0.1 \mathrm{~K})$ described elsewhere $[3,7]$. At standard levels of resolution, two INSTRON systems (Instron 1123 and 5567) were used. Mechanical cycling were performed at a cross-head speed of $20 \mathrm{~mm} / \mathrm{min}$ (Cu-based) and of 10 $\mathrm{mm} / \mathrm{min}$ (NiTi). In order to follow the evolution some representative cycles were recorded at a crosshead speed of $0.1 \mathrm{~mm} / \mathrm{min}$. In realistic behavior with lengthy samples (close to $905 \mathrm{~mm}$ ) a computerized system based on hydraulic displacements are used with maximal frequency near $1 \mathrm{~Hz}$ and available stroke close to $100 \mathrm{~mm}$. The resolution in force, lengthening and temperature are situated near $0.1 \mathrm{~N}, 0.1 \mathrm{~mm}$ and $\pm 2 \mathrm{~K}$ respectively. When necessary a thermocouple type $\mathrm{E}$ or $\mathrm{K}$ with thinner wires (diameter close to $0.08 \mathrm{~mm}$ ) is welded on the sample permitting a direct evaluation of the thermal effects. Transformation temperatures as a function of time and temperature at which the samples were kept have been obtained by means of electrical resistance measurements using a high resolution computerized system $[9,10]$. For resistance measurements diluted nitric acid was used for sample polishing. Cooling and heating were kept at $1.5 \mathrm{~K} / \mathrm{min}$ to allow reliable temperature control and reproducibility.

\section{RESULTS}

The preliminary analysis is centered on the cycling effects on TiNi and CuAlBe (figure 1) and on the evaluation of the Clausius-Clapeyron coefficient. After the classical aging the CuAlBe shows similar effect as the NiTi alloy: an important reduction of the hysteresis width and an increase of the cycle slope. The effect can be associated to higher value of Ms (or As) with partial return to parent phase 
and to some plastic deformation. The effect is clearly diminished with appropriate thermal aging (see, below). The Clausius-Clapeyron coefficients are close to $1.25 \mathrm{MPa} / \mathrm{K}$ for $\mathrm{CuAlBe}$ and to $7.8 \mathrm{MPa} / \mathrm{K}$ for $\mathrm{NiTi}$ (See figure 2 ).
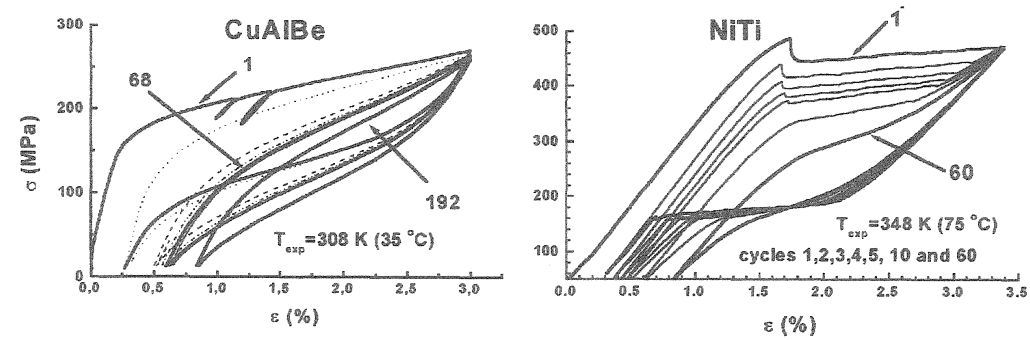

Figure 1: Evolution of the hysteresis cycle with cycling at constant temperature for CuAlBe and NiTi alloys. The hysteresis width changes with cycling from 100 to $50 \mathrm{MPa}$ and from 250 to $75 \mathrm{MPa}$ respectively.
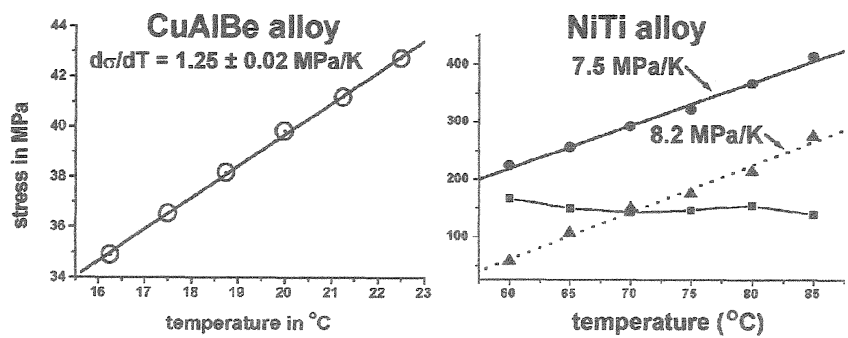

Figure 2: Left: CuAlBe alloy, Clausius Clapeyron coefficient extracted via series of hysteresis cycles performed at $16.25,17.50, \ldots, 22.50^{\circ} \mathrm{C}$. Right: NiTi alloy, evaluation of the Clausius-Clapeyron coefficient; $\bullet \bullet$ critical stress for the transformation curves; $\boldsymbol{\Delta} \boldsymbol{\Delta} \boldsymbol{\Delta}$ critical stress determined from the retransformation path; $\mathbf{\Xi} \boldsymbol{\Xi} \mathbf{m}$ hysteresis width from the same cycles.

The coexistence effects always appears as a complex phenomena with atomic and macroscopic scales. In fast cycles it is usually influenced by thermal effects. Figure 3 shows the behavior of internal loops in CuAlBe alloys at low frequency (near $170 \mathrm{~s} / \mathrm{loop}$ ) at constant deformation. The upper limit of the force increases while the lower value decreases. The figure 4 shows the changes of tha specimen temperature during fast cycling, related to the latent heat and frictional contributions. The effect is highly dependent on the frequency and on the transformation percentage. In the figure 4 the latent heat induces temperature oscillations near $1.5 \mathrm{~K}$ or $6 \mathrm{~K}$ and the asymptotic frictional contributions are close to $1 \mathrm{~K}$ or $4 \mathrm{~K}$ associated to deformations of 2.5 and 5.5 per cent.
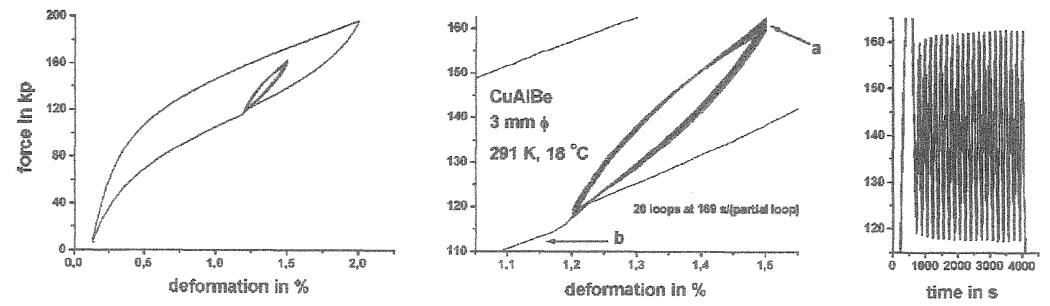

Figure 3: Coexistence effect in CuAlBe alloy via internal loops at low rates, working temperature $301 \mathrm{~K}$. Left: hysteresis cycle with internal loops on unloading. Center: series of internal loops, at constant deformation limits; the $\Delta \mathrm{f}$ increases slighty (see, the arrow a) and the hysteresis is modified (see the arrow b). Rigth: force against time with minor increase at higher value and minor decrease at the lower limit. 
A thermal treatment ensures that the samples start at a reproducible (a well-defined) state. Short time (several minutes) at $1123 \mathrm{~K}$ permits appropriate homogenization of parent phase without relevant changes on grain diameter. Fast quench (in water at room temperature) freezes the bcc phase without precipitation of equilibrium phases. Subsequent aging at near $373 \mathrm{~K}$ improves the quality of the specimen, giving a better behavior of the transformation. Working at "higher room temperatures" the after quenched sample effects on Ms can be visualized. The figure 5 (left) shows the after quench evolution against time for the Ms (CuAlBe polycrystalline sample) determined by the resistance measurements from a temperature induced cycle. Two different aging treatments are used. A classical aging ( $1 \mathrm{~h}$ at $373 \mathrm{~K}$ ) (open dots) and an improved and long time aging (asterisks) at $373 \mathrm{~K}$ and at $293 \mathrm{~K}$. The experimental uncertainty of the Ms value in resistance measurement is close to $\pm 0.5 \mathrm{~K}$. Slow cycling through the martensitic transformation induces small decreases of the Ms temperature (under $3 \mathrm{~K}$ ) as indicated by the arrow (a).

The coexistence effects and the initial creep (as shown in the figure 1) are analyzed after similar aging as indicated in figure 5 left using samples of $905 \mathrm{~mm}$. The figure 5 right shows several series of cycles. Two equal series of cycles at $293 \mathrm{~K}$ and $323 \mathrm{~K}$. (2, 20 and 50 cycles respectively at 0.01 , 0.1 and $0.5 \mathrm{~Hz}$ ). Always with $30 \mathrm{~mm}$ of deformation (close to 3.5 percent). Similar behavior at lower and higher temperatures is observed with negligible permanent plastic deformation. The ClausiusClapeyron coefficient is close to $1.7 \mathrm{~N} / \mathrm{K}$ or $1.2 \mathrm{MPa} / \mathrm{K}$. The values obtained using high resolution stress-strain-temperature equipment [3] furnishes $1.84 \mathrm{~N} / \mathrm{K}$ or $1.25 \mathrm{MPa} / \mathrm{K}$.
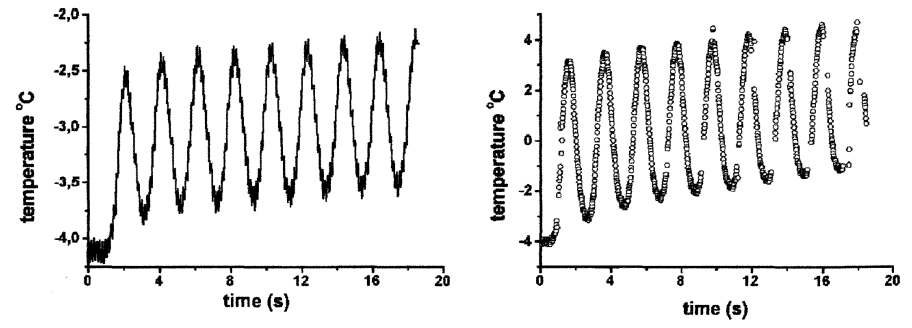

Figure 4: Temperature effects in fast cycling in wire of CuAlBe of $1.44 \mathrm{~mm}^{2}$. The temperature against time is plotted for two transformation percent. Left: $2.5 \%$ and right: $5.5 \%$.
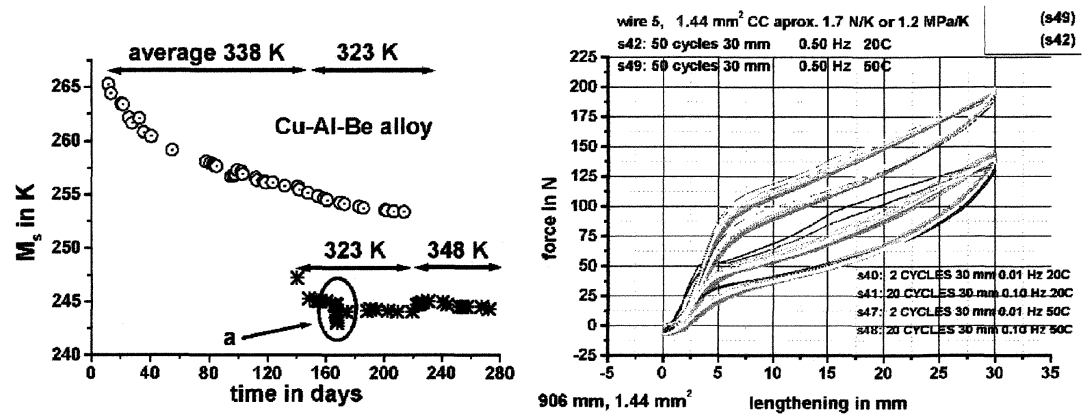

Figure 5: (Left) Transformation temperature (Ms) against time. O O O: Classical Ms evolution after quench with $1 \mathrm{~h}$ of aging at $373 \mathrm{~K}$. "Room temperature" 338 and $323 \mathrm{~K} ; * * *$ Ms minor evolution after long time aging; a: local effects on cycling; after a temperature step (from 323 to $348 \mathrm{~K}$ ) the Ms increases slightly. Right: Sample of $905 \mathrm{~mm}$. Series of cycles realized at 293 and $323 \mathrm{~K}$ : two cycles at $0.01 \mathrm{~Hz}$, twenty cycles at $0.1 \mathrm{~Hz}$ and 50 cycles at $0.5 \mathrm{~Hz}$. The "plastic" permanent deformation is clearly diminished by an increased aging in comparison with figure 1 .

For guaranteed application is always necessary that all transformation temperature fluctuations remain inside bounded levels. From the small step observed in figure 5 left the predictive evolution 
of Ms can be evaluated $[3,8]$. The expected tracking of the yearly fluctuations does not overcome $3 \mathrm{~K}$, and the cycles in figure 5 right and other parallel measurements indicate that the coexistence effects remains under or close to $10 \mathrm{~K}$. The pseudoelastic window needs to include at least: the summer-winter effect (estimated peak to peak fluctuation of $40 \mathrm{~K}$ ), the "after quench" actions (systematic decrease near $5 \mathrm{~K}$ ), the order effects associated to tracking of the seasonal temperature, the coexistence actions inducing a local increase of the Ms $(-10 \mathrm{~K})$, the latent heat and frictional contributions inducing and increase of local temperature near $10 \mathrm{~K}$ (partial transformation in wires with diameter under $3 \mathrm{~mm}$ ) and the hysteresis width (equivalent to $50 \mathrm{~K}$ ). The global effect can be estimated around $90 \mathrm{~K}$ equivalent to $110 \mathrm{MPa}$. In the figure 5-rigth the used domain overcomes $130 \mathrm{MPa}$. The CuAlBe alloys largely satisfy this requirement as they can withstand more than 200 $\mathrm{MPa}$ : in figure 1 left the pseudoelastic window overcomes $250 \mathrm{MPa}$. In a partial analysis performed in the NiTi alloy similar behavior was observed [11], but because of the larger Clausius-Clapeyron coefficient an extremely enlarged pseudoelastic window would be required.

\subsection{Simulation of an earthquake action}

The well established model of the elementary transformation $[3,7-8]$ describes the material behavior in the coordinates force or stress, lengthening and temperature in traction and in 1-dimension actions. As a matter of choice, the model can, eventually, include the time and temperature effects related to microscopic phenomena and the heat transfer actions associated to latent heat and to frictional contributions. Considering the sample divided in $\mathrm{N}$ elements of length $\Delta \mathrm{x}$ and using a well established model of the elementary transformation $[3,7-8]$ is posible to identify the model parameters in single crystals and in polycrystalline samples [3, 11]. From the identification an appropriate simulation is always possible. The figure 6 shows the simulated actions of SMA dampers. The acceleration data from Kyoshin Net (Japan, http://www.k-net.bosai.go.jp/) was used in the computation (station KGS005, earthquake of $13^{\text {th }}$ May 1997, epicenter latitude 31.95; longitude 130.3). The simulated example of figure 6 relates a top slab of mass $7500 \mathrm{~kg}$ (corresponding to $50 \mathrm{~m}^{2}$ at $150 \mathrm{~kg} / \mathrm{m}^{2}$ ). The formalism includes an elastic recovery force term of $10^{6} \mathrm{~N} / \mathrm{m}$, which gives a natural period of $0.54 \mathrm{~s}$. Two SMA dampers working alternatively only in traction composed by 10 bars (diameter: $3 \mathrm{~mm}$ and length: $2.2 \mathrm{~m}$ ) are used. The expected mass of SMA is close to $4.2 \mathrm{~kg}$ when the $x-y$ directions will be damped. The figure shows the quake accelerations, the free oscillation, the damped oscillation and the dissipated energy. Simulation in "winter" shows a slightly better behavior. In fact, the global changes associated to all effects (summer-winter, atomic diffusion and thermal effects) are not relevant for the expected damping.

\section{CONCLUSION}

A careful study of the macroscopic properties (hysteresis cycle, Clausius-Clapeyron coefficient and dynamic contributions by thermal effects) and microscopic actions related with atomic diffusion produced by seasonal effects and by after quenching changes establishes that the working space of $\mathrm{CuAlBe}$ alloy (polycrystalline samples) remains inside the pseudoelastic window (estimated from 0 to $250 \mathrm{MPa}$ ). The local model of the transformation effects and an appropriate identification permit to analyze the alloy behavior under the action of external thermodynamic forces. Simulation of the mechanical accelerations associated with a quake permits an evaluation of the expected alloy behavior in realistic dampers (one-dimensional devices only in traction) built by SMA wires and installed inside a civil structure.

\section{Acknowledgements}

Partial support from European Science Foundation (CONVIB program), MAT2002-02945 (MCYT) and ETSECCPB (Dir. Pol. Territorial) and SGR00152 Gen. Catalonia and is acknowledged. The 
cooperation between CIRG (UPC) and CAB-IB (University of Cuyo, Argentina) is supported by CNEA and DURSI (Gen. Catalonia

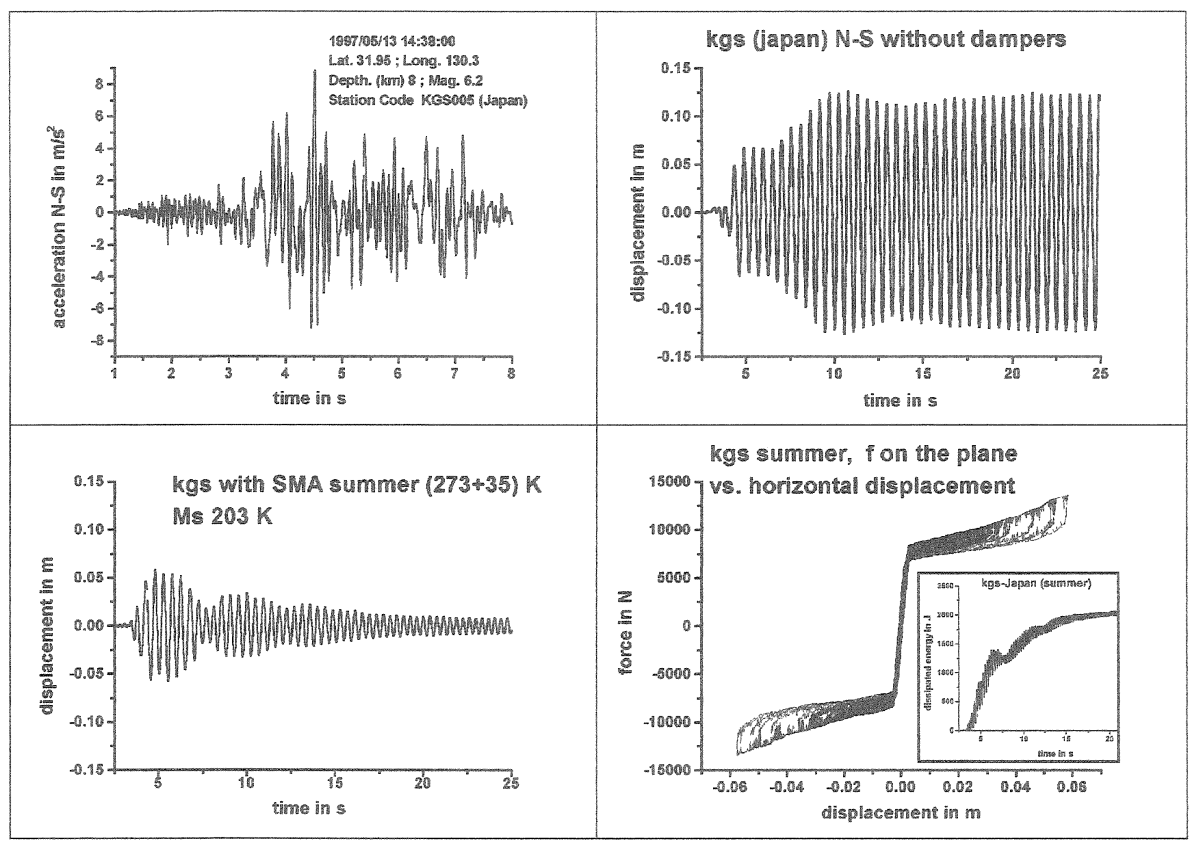

Figure 6: Simulation of the damper actions. From left to rigth and top to bottom: acceleration against time fromm the station KGS005 and the earthquake of $13^{\text {th }}$ May 1997. Free oscillation of slab (data in text). Oscillation with SMA damper (summer). Hysteretic behavior and dissipated energy.

\section{References}

1. M. Ahlers, Prog. Mater. Sci., 30, 135 (1986)

2. K. Otsuka and C. Wayman, Eds. Shape Memory Materials, (Cambridge Univ. Press, UK, 1999)

3. F.C. Lovey, and V. Torra, Prog. Mater. Sci., 44, 189 (1999)

4. V. Torra, Ed. COMETT Course: The Science and Technology of Shape Memory Alloys, (Univ. Illes Balears, Palma de Mallorca, 1989)

5. H. Janocha, Ed. Adaptronics and Smart Structures, (Springer, Berlin, 1999)

6. F. Auricchio, L. Faravelli, G. Magonette and V. Torra, Eds. Shape Memory Alloys. Advances in Modelling and Applications, (CIMNE UPC, Barcelona, 2001)

7. V. Torra, A. Isalgue, F.C. Lovey, M. Sade in Smart Structures and Materials 2002: Smart Systems for Bridges, Structures and Highways, S.-C. Liu, Darryl J. Pines, Editors, Proceedings of SPIE Vol. 4696, p. 186 (2002).

8. V. Torra, A. Isalgue and F.C. Lovey, Materials Transactions of the JIM (special issue on Smart Materials-Fundamentals and Applications), 43 (5), 926 (2002)

9. A. Isalgue and V. Torra, J. Phys. IV, 11, Pr8-141 (2001)

10. A. Amengual, V. Torra, A. Isalgué, and F. Marco, Thermochim. Acta 155, 115 (1989).

11. V. Torra, F. Martorell, A. Isalgue, F.C. Lovey and M. Sade, unpublished results (2003) 